\title{
Does Size Matter? Cryoballoon-Based Pulmonary Vein Isolation Using a Novel 25-mm Circular Mapping Catheter
}

\author{
Bruno Reissmann, MD; Michael Schlüter, PhD; Francesco Santoro, MD; \\ Tilman Maurer, MD; Christian-Hendrik Heeger, MD; Christine Lemes, MD; \\ Thomas Fink, MD; Johannes Riedl, MD; Andreas Rillig, MD; Shibu Mathew, MD; \\ Feifan Ouyang, MD; Karl-Heinz Kuck, MD; Andreas Metzner, MD
}

\begin{abstract}
Background: Real-time recording of pulmonary vein isolation (PVI) using a circular mapping catheter has become a key aspect of cryoballoon (CB) ablation. The aim of this study was to investigate the procedural safety, efficacy and rate of real-time pulmonary vein (PV) recording using a novel circular mapping catheter with a 25-mm loop size for CB-based PVI.
\end{abstract}

\begin{abstract}
Methods and Results: A total of 40 patients with symptomatic atrial fibrillation (AF) underwent PVI using a second-generation CB and a novel 25-mm circular mapping catheter. A total of $159 \mathrm{PV}$ were identified and successfully isolated. Real-time PV recording was achieved in $80 \%$ of the PV. In 3 right inferior PV the circular mapping catheter had to be exchanged for a stiff guidewire due to insufficient mechanical support. Therefore, acute PVI using exclusively the circular mapping catheter was achieved in 156/159 PV (98\%). Mean procedure and fluoroscopy times were $66 \pm 21 \mathrm{~min}$ and $15 \pm 6 \mathrm{~min}$, respectively. Transient phrenic nerve palsy occurred in 1 patient as the only procedural complication.
\end{abstract}

Conclusions: The exclusive use of a novel 25-mm circular mapping catheter for $\mathrm{CB}$ ablation of $\mathrm{AF}$ results in a real-time $\mathrm{PV}$ recording rate of $80 \%$ and isolation of $98 \%$ of targeted PV.

Key Words: Atrial fibrillation; Circular mapping catheter; Cryoballoon ablation; Pulmonary vein isolation; Real-time recording

C ryoballoon $(\mathrm{CB})$ ablation is an effective treatment option for paroxysmal and persistent atrial fibrillation (AF) ${ }^{1-7}$ The procedural endpoint is the electrical isolation of the pulmonary veins (PV) ${ }^{8}$ assessed using circular mapping catheters with loop diameters of 15 or $20 \mathrm{~mm}$ incorporating 8 electrodes (Achieve, Arctic Front Advance, Medtronic, Minneapolis, MN, USA). Initial ablation strategies were based on fixed freeze cycle durations of 180-240 s, frequently also including an added bonus freeze application of the same duration., ,4,9,10 $^{\text {Real-time PV }}$ recordings gained further importance since a correlation of the time to isolation (TTI) of the PV with arrhythmia-free survival was demonstrated. ${ }^{11,12}$ Therefore, novel ablation strategies take the individual TTI into account when setting the total application time, aiming at shorter freeze cycles and thus at reduction of potential complications such as phrenic nerve (PN) palsy and esophageal thermal injury. ${ }^{12-14}$ The consistent application of these TTI-guided ablation protocols, however, is hampered by a limited real-time verification of $\mathrm{PV}$ isolation (PVI) when using currently available 15- or 20-mm diameter circular mapping catheters with 8 electrodes. ${ }^{11-14}$
Recently, a newly designed circular mapping catheter with loop diameters of 15,20 and $25 \mathrm{~mm}$ and a modified solid-core shaft was introduced (Figure 1). The latter is equipped with 10 evenly spaced electrodes (Achieve Advanced $^{\mathrm{TM}}$, Medtronic). The aim of this study was therefore to assess the rate of real-time recordings from inside the PV, acute efficacy, and procedural safety during the routine use of the $25-\mathrm{mm}$ mapping catheter.

\section{Methods}

Inclusion and Exclusion Criteria

Patients with symptomatic paroxysmal or persistent AF were included in the current study. Exclusion criteria were prior left atrial (LA) ablation, LA diameter $>60 \mathrm{~mm}$, severe valvular heart disease or contraindications to post-interventional oral anticoagulation.

\section{Periprocedural Management}

Transesophageal echocardiography was performed prior to PVI in all patients to rule out intracardiac thrombi and to assess the LA diameter. No further pre-procedural

Received September 7, 2017; revised manuscript received November 17, 2017; accepted November 27, 2017; released online December 27, 2017 Time for primary review: 16 days

Department of Cardiology, Asklepios Klinik St. Georg, Hamburg (B.R., F.S., T.M., C.-H.H., C.L., T.F., J.R., A.R., S.M., F.O., K.-H.K., A.M.); Asklepios Proresearch, Hamburg (M.S.), Germany

Mailing address: Bruno Reissmann, MD, Department of Cardiology, Asklepios Klinik St. Georg, Lohmühlenstr. 5, 20099 Hamburg, Germany. E-mail: b.reissmann@asklepios.com

ISSN-1346-9843 All rights are reserved to the Japanese Circulation Society. For permissions, please e-mail: cj@j-circ.or.jp 


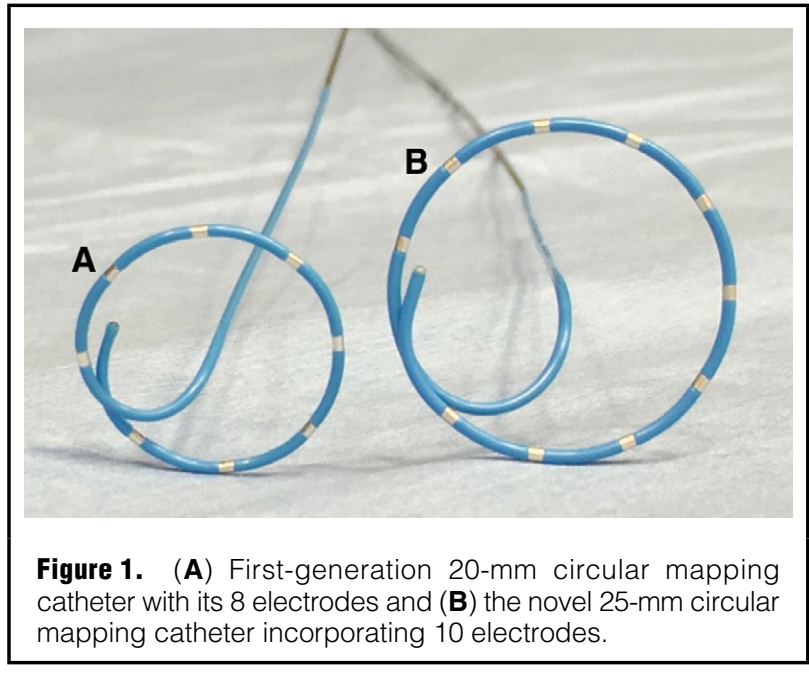

imaging was performed. Ablation was performed under a therapeutic international normalized ratio (INR) of 2-3. Novel oral anticoagulants were stopped the day before the procedure and continued $6 \mathrm{~h}$ after ablation. All patients provided written informed consent. The study is based on a retrospective analysis and was approved by the local ethics board and was performed in accordance with the Declaration of Helsinki.

\section{Ablation}

Patients were placed under deep sedation using i.v. sufentanil, midazolam and propofol. Two 8-F sheaths (St. Jude Medical, Minneapolis, MN, USA) were placed in the right femoral vein. A 6-F steerable decapolar catheter (Inquiry $^{\mathrm{TM}}$, St. Jude Medical) was positioned in the coronary sinus. A single trans-septal puncture was performed via the right femoral vein under fluoroscopy using a modified Brockenbrough technique and an 8.5-F trans-septal sheath (SL1, St. Jude Medical). Heparin was given to maintain an activated clotting time $\geq 300$ s. Selective angiographic visualization by injection of contrast medium was performed to identify the individual PV ostia. The transseptal sheath was then exchanged over a guidewire for a 12-F steerable sheath (FlexCath Advance ${ }^{\mathrm{TM}}$, Medtronic) through which the $28-\mathrm{mm} \mathrm{CB}$ was advanced into the LA. Guiding of the CB to the target PV was performed over a 25-mm inner lumen circular mapping catheter (Achieve Advanced $^{\mathrm{TM}}$, Medtronic), and complete occlusion of the PV ostium was verified by contrast injection through the central lumen of the inflated $\mathrm{CB}$. The circular mapping catheter facilitated real-time verification of PVI by recordings from the $\mathrm{PV}$ ostium, allowing for an ablation strategy taking the individual TTI into account. ${ }^{13}$ When PVI was verified, freezing was continued for an additional fixed period of $120 \mathrm{~s}^{13}$ If the TTI could not be recorded, the freeze cycle duration was set to $180 \mathrm{~s}$. No additional bonus freeze cycle was applied after successful PVI.

An esophageal temperature probe (Sensitherm, St. Jude Medical; or Circa, Circa Scientific) was inserted and positioned according to the individual $\mathrm{CB}$ position to provide esophageal temperature monitoring during energy delivery. The intraluminal esophageal temperature cut-off was set at $15^{\circ} \mathrm{C} .15$

During delivery of cryoenergy along the septal PV,

\begin{tabular}{|lc|}
\hline Table 1. Patient Characteristics & \\
No. patients & 40 \\
Age (years) & $60 \pm 13$ \\
Female & $13(33)$ \\
$\mathrm{BMI}\left(\mathrm{kg} / \mathrm{m}^{2}\right)$ & $28 \pm 5$ \\
LA diameter $(\mathrm{mm})$ & $44 \pm 5$ \\
LVEF $(\%)$ & $55 \pm 4$ \\
$\mathrm{CHA}_{2} \mathrm{DS} \mathrm{S}_{2}$-VASc score & $2(1-2)$ \\
\hline
\end{tabular}

Data given as mean $\pm S D$, median (IQR) or $n(\%)$. BMI, body mass index; LA, left atrium; LVEF, left ventricular ejection fraction.

continuous PN pacing was performed using a diagnostic catheter positioned in the superior vena cava $\left(6 \mathrm{~F}\right.$; Inquiry ${ }^{\mathrm{TM}}$, St. Jude Medical). PN capture was monitored by tactile feedback of diaphragmatic contraction when the operator's hand was placed on the patient's abdomen. In addition, the continuous motor action potential (CMAP) was monitored and refrigerant delivery was stopped immediately if weakening or loss of diaphragmatic movement was noted or the amplitude of the CMAP decreased by $30 \% .{ }^{16,17}$

\section{Post-Procedure Care}

Transthoracic echocardiography was performed in all patients to rule out pericardial effusion. All patients were treated with proton-pump inhibitors for 6 weeks. Lowmolecular-weight heparin was administered to patients on vitamin $\mathrm{K}$ antagonists with INR $<2.0$ until therapeutic INR 2-3 was achieved. Novel oral anticoagulants were reinitiated $6 \mathrm{~h}$ after ablation. Anticoagulation was continued for $\geq 3$ months, and thereafter based on the individual $\mathrm{CHA}_{2} \mathrm{DS}_{2}-\mathrm{VASc}$ score. Previously ineffective anti-arrhythmic drugs were continued for 3 months.

\section{Endpoints}

The primary endpoint was the rate of real-time recordings of CB-based PVI documented by the $25-\mathrm{mm}$ circular mapping catheter. Secondary endpoints included (1) electrical isolation of all PV confirmed on circular-mapping catheter recordings from the PV; (2) procedural parameters (e.g., procedure duration, TTI); and (3) major complications (transient ischemic attack, stroke, pericardial tamponade, PN palsy, and severe bleeding requiring blood transfusion).

\section{Statistical Analysis}

All data were evaluated retrospectively. Continuous data are described as mean \pm SD if normally distributed; otherwise as median (IQR, first quartile; third quartile). Categorical data are described with absolute and relative frequencies. All calculations were performed using SAS (version 9.3, SAS Institute, Cary, NC, USA).

\section{Results}

Patient Characteristics

A total of 40 consecutive patients with symptomatic paroxysmal or persistent AF underwent 28-mm secondgeneration CB-based PVI at Asklepios Klinik St. Georg, Hamburg. In all procedures, the $25-\mathrm{mm}$ circular mapping catheter was used for guidance of the $\mathrm{CB}$ and real-time verification of PVI. Patient baseline characteristics are listed in Table 1. 


\begin{tabular}{|c|c|c|c|c|c|}
\hline & RSPV & RIPV & LSPV & LIPV & LCPV \\
\hline No. PV & 40 & 40 & 39 & 39 & 1 \\
\hline Isolated PV & $40(100)$ & $40(100)^{\dagger}$ & $39(100)$ & $39(100)$ & $1(100)$ \\
\hline No. applications until PVI & $1.1 \pm 0.4$ & $1.5 \pm 1.0$ & $1.3 \pm 0.6$ & $1.2 \pm 0.5$ & 1 \\
\hline Total freeze cycle duration (s) & $149 \pm 27$ & $174 \pm 41$ & $172 \pm 26$ & $159 \pm 25$ & 165 \\
\hline Freeze-cycle duration until confirmed PVI (s) & $25(20-30)$ & $41(29-52)$ & $44(32-50)$ & $29(23-41)$ & 45 \\
\hline No. PVI confirmed in real time & $35(88)$ & $30(75)$ & $28(72)$ & $33(85)$ & $1(100)$ \\
\hline Min. balloon temperature $\left({ }^{\circ} \mathrm{C}\right)$ & $-52 \pm 5$ & $-50 \pm 5$ & $-48 \pm 5$ & $-45 \pm 5$ & -45 \\
\hline Min. endoluminal esophagus temperature $\left({ }^{\circ} \mathrm{C}\right)$ & $35 \pm 1$ & $32 \pm 6$ & $35 \pm 1$ & $34 \pm 2$ & 21 \\
\hline
\end{tabular}

Data given as mean \pm SD, median (IQR) or $n(\%)$. Three RIPV could not be isolated using the $25-\mathrm{mm}$ circular mapping catheter; in each case, exchange of the mapping catheter for a stiff guidewire resulted in successful cryoballoon-based PVI thereafter. LCPV, left common PV; LIPV, left inferior PV; LSPV, left superior PV; PV, pulmonary vein; PVI, PV isolation; RIPV, right inferior PV; RSPV, right superior PV.

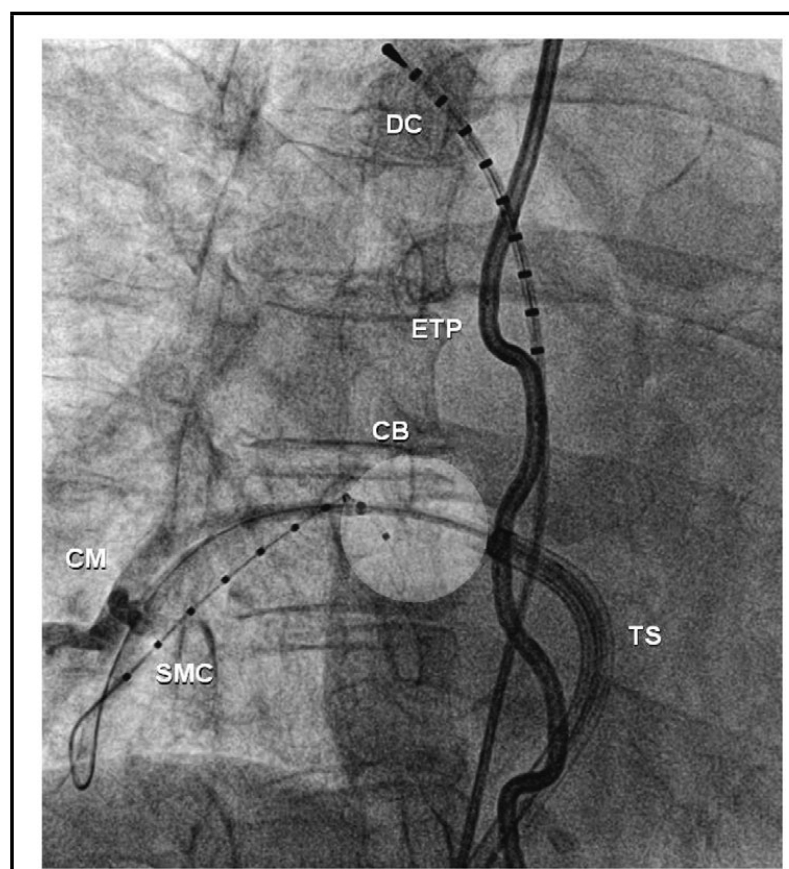

Figure 2. Complete ostial occlusion of the right inferior pulmonary vein (RIPV) is verified by injection of contrast medium (CM) through the central lumen of the inflated cryoballoon (CB). Note the reversed circular mapping catheter (SMC) position. The proximal electrodes are deep inside an inferior branch of the RIPV while the distal electrodes are in an ostial position and in this example trapped in between the balloon surface and the antral pulmonary vein tissue. DC, diagnostic catheter; ETP, esophageal temperature probe; TS, trans-septal sheath. Fluoroscopic right anterior oblique $30^{\circ}$ view.

\section{Procedural Parameters}

In the 40 patients, a total of $159 \mathrm{PV}$ were identified ( 40 right superior PV [RSPV], 40 right inferior PV [RIPV], 39 left superior PV [LSPV], 39 left inferior PV [LIPV] and 1 left common PV [LCPV]; Table 2). All PV were eventually successfully isolated. With the first CB application, electrical PVI was achieved in 38 RSPV (95\%), 30 RIPV (75\%), 28 LSPV $(72 \%)$, 32 LIPV $(82 \%)$, and 1 LCPV $(100 \%)$. The mean number of freeze cycles resulting in PVI was $1.1 \pm 0.3$, $1.5 \pm 1,1.3 \pm 0.6,1.2 \pm 0.5$ and 1 for the RSPV, RIPV, LSPV,
LIPV, and LCPV, respectively. Real-time recording of PVI was achieved in 35 RSPV (88\%), 30 RIPV (75\%), 28 LSPV $(72 \%), 33$ LIPV (85\%), and in 1 LCPV $(100 \%)$. The overall rate of real-time PV recordings was $80 \%(95 \% \mathrm{CI}$ : 73-86\%).

Three RIPV, each characterized by a low insertion level into the LA, could not be ablated and isolated using the 25-mm mapping catheter due to incomplete PV occlusion despite multiple balloon and mapping catheter maneuvers and despite different sheath angulations. Even when aiming at maximum mechanical support by forgoing real-time electrical recordings but positioning the mapping catheter at distal levels of the target PV, occlusion was never perfect. The mapping catheter was then replaced for a stiff guidewire (Amplatz Super Stiff ${ }^{\mathrm{TM}}$ Guidewire, Boston Scientific, Plymouth, MN, USA) aiming at more mechanical support. In each of these cases, successful CB-based PVI was then achieved. Thus, the rate of PVI achieved using exclusively the 25-mm mapping catheter was 156/159 (98\%; 95\% CI: $95-100 \%)$.

Median freeze cycle duration until real-time verification of PVI was $25 \mathrm{~s}$ (IQR, 20-30s) for RSPV, 41 s (IQR, 29-52s) for RIPV, 44s (IQR, 32-50s) for LSPV, 29s (IQR, 23-41 s) for LIPV, and $45 \mathrm{~s}$ for the LCPV (Table 2). Mean total freeze cycle duration including PV without real-time signal recordings and fixed freeze cycle durations of $180 \mathrm{~s}$ was $149 \pm 27 \mathrm{~s}, 174 \pm 41 \mathrm{~s}, 172 \pm 26 \mathrm{~s}, 159 \pm 25 \mathrm{~s}$, and $165 \mathrm{~s}$ for the RSPV, RIPV, LSPV, LIPV and LCPV, respectively. Mean minimum balloon temperature and mean minimum endoluminal esophageal temperature for the respective PV are also given in Table 2. Mean procedure and fluoroscopy times were $66 \pm 21 \mathrm{~min}$ and $15 \pm 6 \mathrm{~min}$, respectively. The median dose area product was $637 \mathrm{cGycm}^{2}$ (IQR, 436$\left.929 \mathrm{cGycm}^{2}\right)$.

\section{5-mm Circular Mapping Catheter: Special Aspects}

In most PV the $25-\mathrm{mm}$ circular mapping catheter can be easily advanced into the target PV and positioned in close proximity to the balloon surface in its normal circular shape, thereby providing signals from all 10 electrodes. In contrast, when the 25-mm mapping catheter is used in small PV or in PV with multiple proximal branches, insertion of the catheter into one of these branches often resulted in a characteristic reversed circular mapping catheter position (Figure 2). In this position, the proximal electrodes were deep inside the PV and the distal electrodes close to the PV orifice, allowing for efficient mechanical support and resulting in PV signal recordings only from 


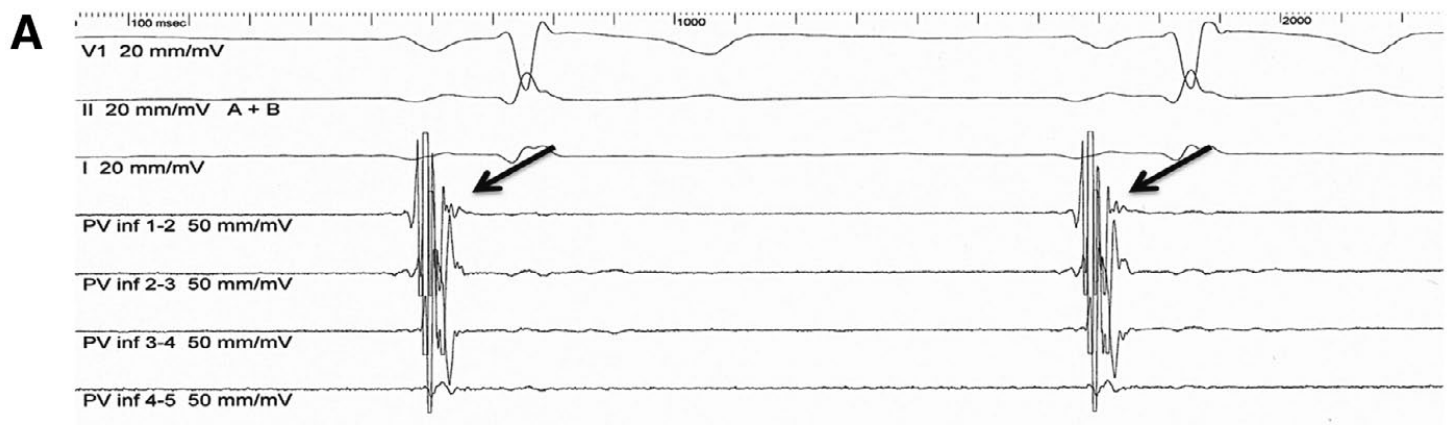

$\widehat{P V}$ inf $5-650 \mathrm{~mm} / \mathrm{mV}$

PV inf 6-7 $50 \mathrm{~mm} / \mathrm{mV}$

PV inf $7-850 \mathrm{~mm} / \mathrm{mV}$

PV inf 8-9 $50 \mathrm{~mm} / \mathrm{mV}$

PV inf $9-1050 \mathrm{~mm} / \mathrm{mV}$

CS $1-210 \mathrm{~mm} / \mathrm{mV}[\mathrm{CH} 1$

B
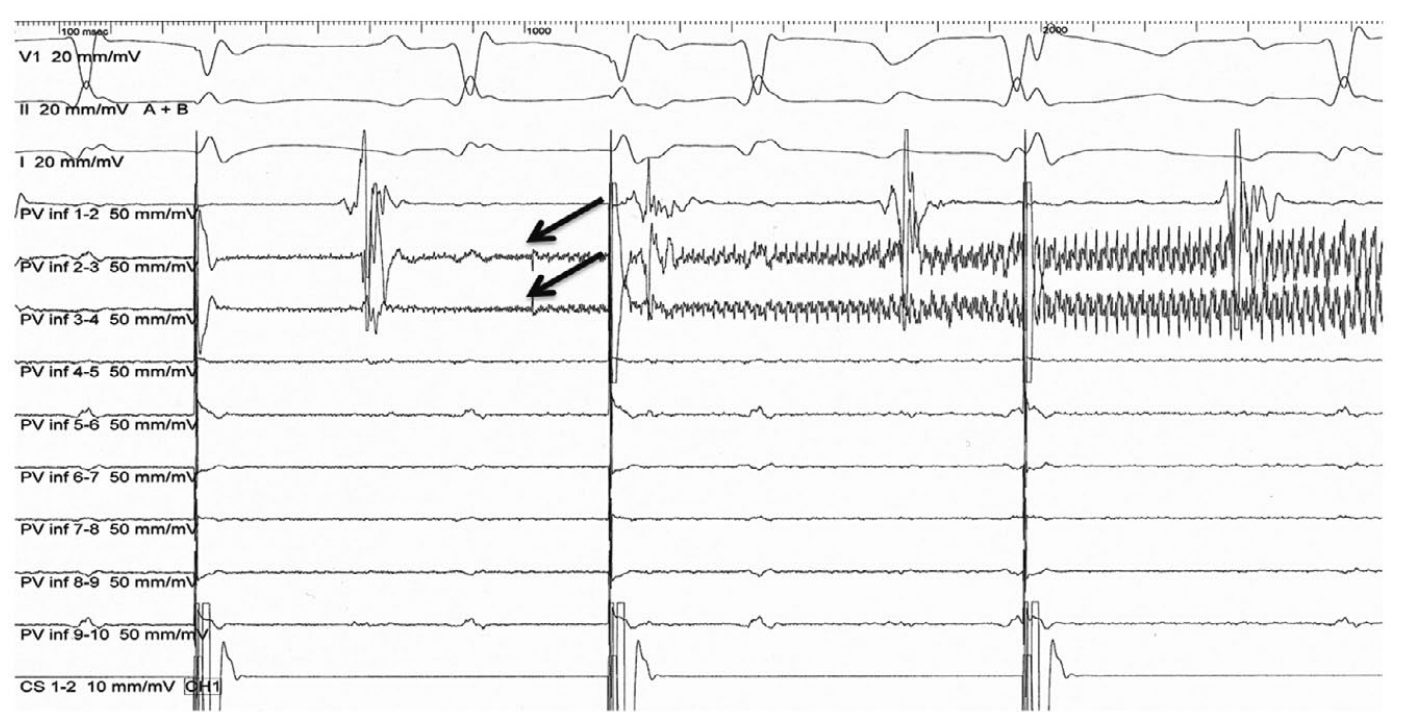

Figure 3. (A) Tracing of surface electrocardiogram (ECG) leads I, II, and V1 and intracardiac electrograms recorded from the 10 electrodes of the 25-mm circular mapping catheter (PV inf 1-10) within the right inferior pulmonary vein (RIPV; cf. Figure 2). A diagnostic catheter is positioned in the superior vena cava to perform continuous phrenic nerve pacing (CS 1-2). Real-time signals from the circular mapping catheter are recorded only by distal electrodes (PV inf 1-2 and PV inf 3-4; arrows), which are in an antral position, whereas the proximal electrodes are deep inside the RIPV. The electrograms from the proximal electrodes reflect fusion of far-field atrial and near-field PV potentials. (B) Surface ECG leads I, II, and V1 and intracardiac electrograms recorded from the 10 electrodes of the 25-mm circular mapping catheter (PV inf 1-10) positioned within the right inferior pulmonary vein (cf. Figure 1). Continuous phrenic nerve pacing is performed by a diagnostic catheter positioned in the superior vena cava (CS 1-2). The close proximity of the distal circular mapping catheter electrodes (PV inf 1-2 and PV inf 3-4) to the surface of the cryoballoon results in freezing artifacts starting at $24 \mathrm{~s}$ of the freeze cycle, thus preventing a clear assessment of real-time recording of $\mathrm{PV}$ isolation (arrows).

the distal electrodes. In some of these cases, however, the distal electrodes of the mapping catheter were trapped between the balloon surface and the antral PV tissue, making the real-time differentiation between far-field atrial and PV potentials more challenging (Figures 2,3A). Additionally, the close proximity of these electrodes to the CB surface resulted in freezing artifacts in an early phase of the respective freeze cycle, thereby preventing a clear real-time assessment of PVI (Figure 3B).

\section{Complications}

Transient PN palsy was observed in 1 patient during CB ablation along the RSPV; at that time, the RSPV had already been successfully isolated. Freezing was immediately 
stopped and the $\mathrm{PN}$ recovered within $30 \mathrm{~min}$. No further complications occurred.

\section{Discussion}

The current study is the first to report procedural characteristics using a novel $25-\mathrm{mm}$ circular mapping catheter routinely for $\mathrm{CB}$ ablation of AF. Major findings are (1) rate of real-time $\mathrm{PV}$ recording of $80 \%$; (2) high acute success rates; and (3) high procedural safety.

\section{Real-Time PV Recording}

The first-generation inner lumen circular mapping catheter allows for over-the-wire support of the balloon, real-time $\mathrm{PV}$ recordings, and assessment of PVI. Currently the CB in combination with a circular mapping catheter is the only balloon-based ablation system that allows for routine and systematic real-time assessment of PV signals. First studies evaluating mapping technologies in conjunction with the hot-balloon, however, are currently under investigation. The TTI independently predicts recovered PV-LA conduction $^{11}$ and thereby directly affects arrhythmiafree survival. ${ }^{12,18}$ The direct feedback on freeze cycle efficacy enables the operator to anticipate ineffective freeze cycles, which should result in premature discontinuation of the respective application. In contrast, challenging variants of PV or LA anatomies might prevent PVI at an early stage of the freeze cycle; in this setting, freeze cycle durations of $180 \mathrm{~s}$ or $240 \mathrm{~s}$ might be too short to ensure durable PVI, and individualized freeze cycle durations $>180$ s or 240 s might be necessary. Therefore, an increased incidence of real-time PV recordings will facilitate individualized ablation strategies and might further enhance the efficacy and safety of CB-based PVI. ${ }^{\mathbf{1 2}, 13}$

The rate of real-time recordings using the first-generation inner lumen circular mapping catheter was in the range of $40-76 \% .^{13,19}$ In the current study, the use of the novel second-generation $25-\mathrm{mm}$ circular mapping catheter resulted in a rate of real-time PV recordings of $80 \%$. In 3 of the present patients, however, the circular mapping catheter had to be exchanged for a stiff guidewire after failed attempts at adequate PV occlusion due to insufficient mechanical support. The use of a stiff guidewire then resulted in complete PV occlusion and successful PVI in all 3 patients. This is in contrast to previous publications describing successful isolation of all targeted PV using exclusively the first-generation 20-mm circular mapping catheter. ${ }^{13,14,19}$ The requirement to replace the $25-\mathrm{mm}$ mapping catheter in these patients might be attributed to the new solid-core shaft design of the catheter, which appears to be less stiff than the previous first-generation circular mapping catheter; it thereby potentially offers less mechanical support in certain anatomical settings, for example, a low insertion level of the RIPV into the LA and early branching in close proximity to the LA-PV junction. In this particular setting, we think that the stiffer shaft of the first-generation Achieve catheter provides better mechanical support and stability than the new design shaft of the second-generation Achieve catheter. All other PV were successfully isolated using standard CB maneuvers.

\section{Learning Curve}

The fluoroscopy times in the current study are longer compared with a previous publication on $\mathrm{CB}$ ablation from our group. ${ }^{14}$ The performance, however, of the new circular mapping catheter with its new shaft design is different to the previous catheter generation, and requires a certain learning curve even when procedures are performed by experienced operators.

\section{Study Limitations}

The current study is an observational, non-randomized single-center analysis with a limited number of patients, and more experience is required before final conclusions on the new circular mapping catheter generation can be drawn.

\section{Conclusions}

The exclusive use in a routine clinical setting of a novel 25-mm circular mapping catheter for $\mathrm{CB}$ ablation of $\mathrm{AF}$ resulted in a real-time PV recording rate of $80 \%$ and isolation of $98 \%$ of targeted PV. TTI-guided ablation protocols might be effectively supported.

\section{Acknowledgments}

None.

\section{Disclosures}

B.R. and C.-H.H. received travel grants from Medtronic. A.M. received speaker honoraria and travel grants from Medtronic. K.-H.K. received research grants from Medtronic, and is a consultant for Medtronic. The other authors report no conflicts of interest.

\section{References}

1. Kuck KH, Brugada J, Furnkranz A, Metzner A, Ouyang F, Chun KR, et al. Cryoballoon or radiofrequency ablation for paroxysmal atrial fibrillation. $N$ Engl J Med 2016; 374: $2235-$ 2245.

2. Kuck KH, Furnkranz A, Chun KR, Metzner A, Ouyang F, Schluter M, et al. Cryoballoon or radiofrequency ablation for symptomatic paroxysmal atrial fibrillation: Reintervention, rehospitalization, and quality-of-life outcomes in the FIRE AND ICE trial. Eur Heart $J$ 2016; 37: 2858-2865.

3. Luik A, Radzewitz A, Kieser M, Walter M, Bramlage P, Hormann P, et al. Cryoballoon versus open irrigated radiofrequency ablation in patients with paroxysmal atrial fibrillation: The prospective, randomized, controlled, noninferiority FreezeAF Study. Circulation 2015; 132: 1311-1319.

4. Metzner A, Reissmann B, Rausch P, Mathew S, Wohlmuth P, Tilz R, et al. One-year clinical outcome after pulmonary vein isolation using the second-generation $28-\mathrm{mm}$ cryoballoon. Circ Arrhythm Electrophysiol 2014; 7: 288-292.

5. Tscholl V, Lsharaf AK, Lin T, Bellmann B, Biewener S, Nagel $\mathrm{P}$, et al. Two years outcome in patients with persistent atrial fibrillation after pulmonary vein isolation using the secondgeneration 28-mm cryoballoon. Heart Rhythm 2016; 13: 1817 1822.

6. Koektuerk B, Yorgun H, Hengeoez O, Turan CH, Dahmen A, Yang A, et al. Cryoballoon ablation for pulmonary vein isolation in patients with persistent atrial fibrillation: One-year outcome using second generation cryoballoon. Circ Arrhythm Electrophysiol 2015; 8: $1073-1079$.

7. Lemes C, Wissner E, Lin T, Mathew S, Deiss S, Rillig A, et al. One-year clinical outcome after pulmonary vein isolation in persistent atrial fibrillation using the second-generation $28 \mathrm{~mm}$ cryoballoon: A retrospective analysis. Europace 2016; 18: $201-$ 205.

8. Kirchhof P, Benussi S, Kotecha D, Ahlsson A, Atar D, Casadei $\mathrm{B}$, et al. 2016 ESC Guidelines for the management of atrial fibrillation developed in collaboration with EACTS. Eur Heart $J$ 2016; 37: 2893-2962.

9. Chierchia GB, Di Giovanni G, Ciconte G, de Asmundis C, Conte G, Sieira-Moret J, et al. Second-generation cryoballoon ablation for paroxysmal atrial fibrillation: 1-year follow-up. Europace 2014; 16: 639-644.

10. Ciconte G, de Asmundis C, Sieira J, Conte G, Di Giovanni G, 
Mugnai G, et al. Single 3-minute freeze for second-generation cryoballoon ablation: One-year follow-up after pulmonary vein isolation. Heart Rhythm 2015; 12: 673-680.

11. Ciconte G, Mugnai G, Sieira J, Velagic V, Saitoh Y, Irfan G, et al. On the quest for the best freeze: Predictors of late pulmonary vein reconnections after second-generation cryoballoon ablation. Circ Arrhythm Electrophysiol 2015; 8: 1359-1365.

12. Chun KR, Stich M, Furnkranz A, Bordignon S, Perrotta L, Dugo $\mathrm{D}$, et al. Individualized cryoballoon energy pulmonary vein isolation guided by real-time pulmonary vein recordings, the randomized ICE-T trial. Heart Rhythm 2017; 14: 495-500.

13. Reissmann B, Wissner E, Deiss S, Heeger C, Schlueter M, Wohlmuth P, et al. First insights into cryoballoon-based pulmonary vein isolation taking the individual time-to-isolation into account. Europace 2017; 19: 1676-1680.

14. Reissmann B, Maurer T, Wohlmuth P, Kruger M, Heeger C, Lemes $C$, et al. Significant reduction of radiation exposure in cryoballoon-based pulmonary vein isolation. Europace, doi: 10.1093/europace/eux066.

15. Furnkranz A, Bordignon S, Bohmig M, Konstantinou A, Dugo $\mathrm{D}$, Perrotta L, et al. Reduced incidence of esophageal lesions by luminal esophageal temperature-guided second-generation cryoballoon ablation. Heart Rhythm 2015; 12: 268-274.

16. Metzner A, Rausch P, Lemes C, Reissmann B, Bardyszewski A, Tilz $\mathrm{R}$, et al. The incidence of phrenic nerve injury during pulmonary vein isolation using the second-generation $28 \mathrm{~mm}$ cryoballoon. J Cardiovasc Electrophysiol 2014; 25: 466-470.

17. Mondesert B, Andrade JG, Khairy P, Guerra PG, Dyrda K, Macle L, et al. Clinical experience with a novel electromyographic approach to preventing phrenic nerve injury during cryoballoon ablation in atrial fibrillation. Circ Arrhythm Electrophysiol 2014; 7: 605-611.

18. Ouyang F, Antz M, Ernst S, Hachiya H, Mavrakis H, Deger FT, et al. Recovered pulmonary vein conduction as a dominant factor for recurrent atrial tachyarrhythmias after complete circular isolation of the pulmonary veins: Lessons from double Lasso technique. Circulation 2005; 111: 127-135.

19. Heeger CH, Wissner E, Mathew S, Hayashi K, Sohns C, Reissmann B, et al. Short tip-big difference?: First-in-man experience and procedural efficacy of pulmonary vein isolation using the third-generation cryoballoon. Clin Res Cardiol 2016; 105: $482-488$. 